\title{
Disease Watch
}

\section{EBOLA CRISIS CONTINUES}

Outbreak update. The ongoing epidemic of Ebola virus (EBOV), which has infected more than 13,000 people and has claimed approximately 5,000 lives so far, is now showing the first signs of regression in Liberia, the worst affected country in West Africa. The WHO has also reported an end to the outbreak in Nigeria but, worryingly, the first case of EBOV was confirmed in Mali in October. The WHO cautions that the crisis is far from over and predicts that, by December 2014, up to 10,000 new cases could emerge per week if the outbreak is not adequately controlled. WHO/BBC News

Medical interventions. Without an approved vaccine or drugs, management of the disease is a major challenge. Supplies of the experimental drug ZMapp (comprising three monoclonal antibodies that target EBOV envelope proteins) have now run out, but efforts to fast-track the testing of vaccines, other experimental drugs and purified plasma from EBOV survivors, are underway. Dosing and safety trials in humans for the first two vaccines (which have been developed by GlaxoSmithKline and Johnson \& Johnson) are due to begin in December 2014, and a third vaccine (developed by the Public Health Agency of Canada) is likely to be accelerated through to human safety trials following the announcement of $£ 3.1$ million funding by the Wellcome Trust. The WHO are coordinating the trials and aim to deploy the vaccines as soon as possible during the first half of 2015. A recent study by Pandey, Atkins et al. evaluated the value of non-pharmaceutical interventions for curbing the epidemic in Liberia, and reported that a combined approach of case isolation, contact tracing with quarantine and sanitary funeral practices require urgent implementation to halt the outbreak in West Africa, all of which require substantial international aid. WHO/The Guardian

Viral pathogenesis. Currently available mouse models for EBOV do not develop the hallmark symptoms of Ebola virus disease, such as delayed blood coagulation and disseminated intravascular haemorrhaging, so pathogenesis studies have been limited to non-human primates, which are impractical for large-scale studies. However, Rasmussen, Okumura et al. now report the use of a genetically diverse panel of recombinant inbred mice (known as Collaborative Cross mice) and show that they reproduce the range of pathological manisfestations experienced by humans (from complete resistance to mild and lethal disease) following infection with mouse-adapted EBOV. Interestingly, the authors find that host genetic factors are a major contributor to the severity of infection. For example, expression of the endothelial tyrosine kinase TEK, which activates coagulation factors, was consistently high in the spleen of mice resistant to lethal infection, which suggests that blood vessel repair and subsequent control of vascular leakage are important for ensuring survival. As this approach can map susceptibility alleles, it has the potential to assist future drug and vaccine development efforts.

ORIGINAL RESEARCH PAPERS Pandey, A., Atkins, K. E. et al. Strategies for containing Ebola in West Africa. Science http://dx.doi.org/10.1126/science.1260612 (2014) | Rasmussen, A. L., Okumura, A. et al. Host genetic diversity enables Ebola hemorrhagic fever pathogenesis and resistance. Science http://dx.doi.org/10.1126/science.1259595 (2014) 